\title{
A Novel Approach for Efficient Route Request Flooding in MANETs with Directional Antennas
}

\author{
Long Qian, Huyin Zhang, Di Wu, Jing Wang \\ School of Computer \\ Wuhan University \\ Wuhan City, Hubei Province, China \\ channel@whu.edu.cn
}

\begin{abstract}
In recent years, using directional antennas to reduce the amount of interference and collisions in mobile ad hoc networks has become more and more popular. Due to the problem of broadcast storm caused by flooding route requests, a novel route request flooding approach called Hexagonal Flooding Strategy (HFS) for Ad Hoc networks using directional antennas is introduced in this paper. With directional antennas, the flooding of route request packets is limited among selected forwarding nodes. By constructing a honeycomb pattern, the forwarding nodes which form the honeycomb vertices are selected. The transmissions between forwarding nodes which form the honeycomb edges are reshaped according to certain rules to optimize flow directions. Through simulations we evaluate the performance of HFS using route discovery success rate, average number of nodes and forwardings involved in the route discovery process. Simulation results show that HFS can: (a) improve route discovery success rate, (b) reduce the number of nodes and forwardings involved in the route discovery process.
\end{abstract}

Keywords—Ad Hoc Network; Broadcast storm; Directional Antennas; Route Request Flooding; Honeycomb Strategy

\section{INTRODUCTION}

Mobile Ad Hoc network (MANET) is a multi-hop mobile wireless network which does not have any preexisting network infrastructure or centralized administration $^{[1]}$. Due to its convenience of mobile communication, MANET is explored for numerous applications, such as network extension, ubiquitous computing, urban sensing and vehicular networking. Using directional antennas to reduce the amount of interference and collisions in mobile ad hoc networks has become more and more popular in recent years. Directional antennas can significantly increase the spatial reuse and have longer transmission distance without spending extra power due to their high gain. They can reduce energy consumption and prolong the network lifetime ${ }^{[2]}$.

A wide range of routing protocols for MANET have been developed, which can be categorized into table-driven and on-demand routing protocols. Most of on-demand routing protocols perform route discovery, during which route request packets are flooded throughout the network ${ }^{[3]}$. The broadcast storm caused by flooding route requests causes a lot of unnecessary overhead. Directional antennas are also used to enhance the route request flooding mechanism. Route request flooding is different from real directional broadcast protocol whose goal is that each node in the system receives a broadcast message at least. As long as the routing protocol can effectively find the route, route request flooding does not need broadcast messages to be received by all nodes. Therefore, the flooding of route request packets can be limited among selected forwarding nodes. Through the rational design of selection strategy of forwarding nodes, the redundancy of route request packets can be reduced greatly.

In this paper, we propose a novel route request flooding approach called Hexagonal Flooding Strategy (HFS) for Ad Hoc networks using directional antennas. By constructing a honeycomb pattern, the forwarding nodes which form the honeycomb vertices are selected. The transmissions between forwarding nodes which form the honeycomb edges are reshaped according to certain rules to optimize flow directions. Route request packets are forwarded from source node to the honeycomb vertices along honeycomb vertices. The main idea of the proposed route request flooding approach is to strategically select a small subset of nodes as forwarding nodes to forward route request packets. It is shown from the simulation results that compared with the existing approaches, HFS can improve route discovery success rate, and reduce the number of nodes and forwardings involved in the route discovery process simultaneously.

The remainder of this paper is organized as follows: Section 2 describes the previous studies on directional broadcast protocols and route request flooding mechanism. In Sections 3, we introduce antennas model and network model. The proposed selection strategy of forwarding nodes and route request flooding algorithm are present in Sections 4. In addition, the simulation results are presented and analysed in Section 5. Then we conclude the paper in Section 6 .

\section{RELATED WORK}

Over the years, many approaches have been proposed to reduce the the redundancy of route request packets. Several approaches where each node forwards route request packets with some probability were proposed to reduce the flooding overhead, while several route discovery protocols using directional antennas were proposed. In addition, a lot of approaches give their unique solutions to solve this problem.

Aminu Mohammed et al. ${ }^{[4]}$ proposed a new probabilistic counter-based method which combine the 
features of counter-based and gossip-based approaches. The proposed method can significantly reduce the number of RREQ packets transmitted during route discovery operation. Similarly, in [5] the authors proposed a probabilistic method of flooding that adjusts the forwarding probability at a node using its local topological characteristics. Unlike the first two, Li Jian et al. ${ }^{[6]}$ proposed a Positional Attribute based Next-hop Determination Approach and identified the "next-hop racing" phenomena due to the random rebroadcast delay approach during the route discovery process. The proposed approach can effectively avoid the problem of wireless broadcast storm.

A number of directional antennas based route discovery protocols have been proposed for wireless ad hoc networks. Yongsuk Park and Taejoon Park ${ }^{[7]}$ present a route discovery protocol for ad hoc multi-hop cellular networks which uses directional information towards the base station. The proposed protocol, based on the reactive approach, reduces flooding as much as possible. In [8], Marco Aur é lio Spohn et al. apply two enhancements aimed at reducing the overhead incurred in the dissemination of RREQs. A distributed connected dominating-set algorithm is used to reduce the number of nodes that need to propagate RREQs transmitted on broadcast mode, and information regarding prior routes to a destination is used to unicast RREQs to a region close to the intended destination. Rully Adrian Santosa et al. ${ }^{[9]}$ proposed an efficient scheme for flooding the DSR route request in ad hoc networks using directional antennas. Fixed branching and honeycomb strategies are proposed to efficiently select the representative neighbors to forward route request packets, which are able to achieve a high route discovery success rate while keeping the number of involved nodes and the number of transmissions low.

There are also a lot of approaches give their unique solutions. Bani Khalaf $M$. et al. ${ }^{[10]}$ proposed a new distributed route discovery scheme to handle the broadcast operation efficiently by reducing the number of the broadcast redundancy request packets and the number of collision and contentions. In [11], Lukas Wallentin et al. proposed a new cross layer optimized route discovery scheme using a distributed route pre-selection technique in combination with artificial delaying of route discovery packets. In [12], Natarajan Meghanathan present a novel network density and mobility aware energy-efficient broadcast route discovery strategy to determine stable routes in MANETs. The proposed strategy is very effective in reducing the number of route request packets. Xiangyang Jin et al. ${ }^{[13]}$ an On-demand Tree-based Routing Protocol for ad hoc networks which use an efficient route discovery algorithm called Tree-based Optimised Flooding to reduce the number of consumed RREQ retries.

\section{PRELIMINARIES}

\section{A. Antennas model}

Directional antennas are a kind of antennas which has strong transmitting and receiving electromagnetic waves particularly in one or a few specific direction, while weak electromagnetic waves in other directions. Directional antennas can concentrate transmission power in a particular direction, thereby providing a longer transmission range. At the same time, directional antennas can localize signal interference, reduce routing hops, and increase network life to reduce the occurrence of network partition. By increasing the potential for spatial reuse, directional antennas can improve the network capacity.

The proposed antennas model is comprised of six directional antennas, which have conical radiation pattern, spanning the angle of $\pi / 3$. As shown in Figure 1 , the numbers of directional antennas increase in clockwise order, are $0,1,2,3,4$, and 5 , respectively. If antenna number is $m$, the numbers of the two adjacent antennas are $(m+1) \% 6$ and $(m-1) \% 6$, wherein the number of clockwise adjacent antenna is $(m+1) \% 6$, the number of counterclockwise adjacent antenna is $(m-1) \% 6$.

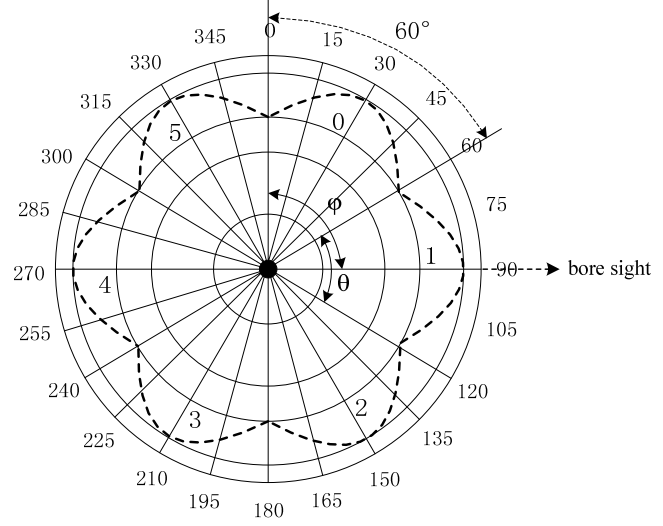

Figure 1. The radiation pattern of antenna system

With directional antennas, the transmission range of each node is equally divided into six non-overlapping sectors. The antenna orientation $\varphi(0 \leq \varphi \leq 2 \pi)$ of node is defined as the angle measured clockwise from the vertical axis to the antenna bore sight, and the antenna beamwidth $\theta(0 \leq \theta \leq 2 \pi)$ is defined as the angle of beamwidth. Each node can use any antenna for communication at any time.

\section{B. Network model}

We make the following assumptions to the network model: each node is equipped with directional antennas following the proposed antennas model; the irregular shapes of antenna beams due to the existence of side lobes are ignored; each node knows the location of the neighbors and its own location. As shown in Figure 2, the location information of neighbor node $\mathrm{A}$ includes:

- Neighbor identity $i$, which is the identity of A;

- Antenna number $m$, which is the number of directional antenna communicates with $\mathrm{A}$;

- Neighbor angle $a(-\pi / 6 \leq a \leq \pi / 6)$, which is the angle measured clockwise from the direction of $\mathrm{A}$ to the antenna bore sight;

- Neighbor distance $d$, which is distance between source node to A;

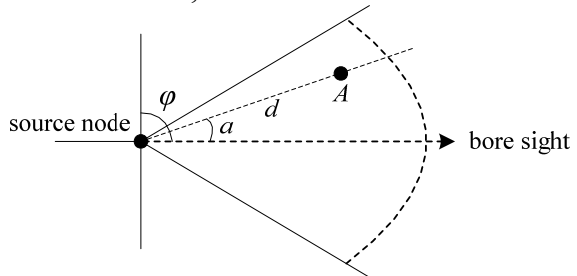

Figure 2. The propagation model of antenna system 
Directional neighborhood discovery provides a node with the location information of neighbor node around it via exchanging "Hello" messages. Each node sends "Hello" messages to its neighbor nodes in all directions periodically. The identity and location of nodes are piggybacked in the messages. With the location of neighbor nodes collected and its own position, each node can calculate neighbor angle and neighbor distance, then assemble its location information of 1-hop neighbor node. By exchanging location information of 1-hop neighbor node via "Hello" messages, node can assemble its location information of 2-hop neighbor node.

\section{HeXagonal Flooding StRategy}

\section{A. Overview}

In traditional route discovery, route request packets are forwarded to each node in the network as much as possible, like a broadcast protocol. As long as route can be efficiently discovered, route discovery does not need route request packets to be received by all nodes. When all nodes have the information of its neighbors, route discovery process can be terminated at last-hop of the destination node instead of destination node itself.

In the proposed strategy, the flooding of route request packets is limited among selected forwarding nodes. Forwarding nodes are special subset of all nodes, which have the following characteristics. Firstly, the union of the neighbor nodes of all forwarding nodes should cover all the nodes. Secondly, the number of forwarding nodes should be as small as possible. Finally, the distribution of forwarding nodes in the space should be as uniform as possible. Based on the above requirements, in an ideal state, each node in the system appears in the neighbor list of one and only one forwarding node. By constructing a honeycomb pattern, the nodes form the honeycomb vertices are selected as forwarding nodes which can satisfy the above points well.

Similarly, the broadcast storm caused by flooding route requests among forwarding nodes will also cause unnecessary redundancy. With directional antennas, the transmissions between forwarding nodes are reshaped according to certain rules to reduce unnecessary forwarding. When a source node initiates the route discovery process, it checks its neighbor list to find the destination node first. If the destination node is not one of its neighbor nodes, it selects forwarding nodes and forwards route requests to them according to the proposed strategy. The forwarding node having the destination node as its neighbor will send route reply packet to source node, then the route has just been discovered.

\section{B. Selection of forwarding nodes}

In Hexagonal Flooding Strategy, the forwarding node is selected as the neighbor node which is the farthest from the node in the predefined area. As shown in Figure 3, for each antenna, a sector with a fixed width is defined as predefined area. The sector is evenly distributed on both sides of the antenna bore sight, and the width of the sector is a small angle which is denoted by $\varepsilon$. The neighbor node farthest from the source node that falls in the predefined area is selected as the forwarding node. Because the proposed antennas model is comprised of six directional antennas, each node selects six neighbor nodes as their forwarding nodes. The selection of forwarding nodes is presented in Algorithm 1.

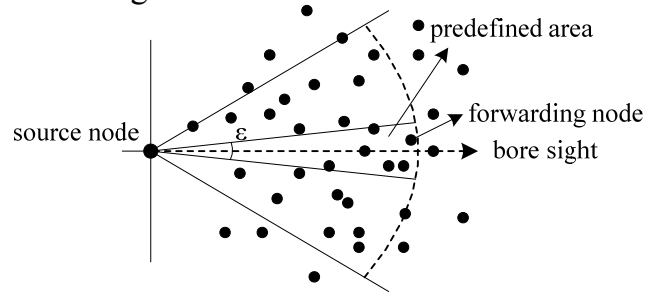

Figure 3. The selection of a forwarding node

\begin{tabular}{ll}
\hline \multicolumn{2}{l}{ Algorithm 1. The selection of forwarding nodes } \\
\hline 1 & let $M$ be the list of antennas of source node \\
2 & let $F$ be the list of forwarding nodes \\
3 & let $F=N U L L$ \\
4 & for all $m$ in $M$ do \\
5 & let $N$ be the neighbor list of source node \\
6 & for all $n$ in $N$ do \\
7 & let $m_{n}$ be the antenna number of node $n$ \\
8 & let $a_{n}$ be the neighbor angle of node $n$ \\
9 & let $d_{n}$ be the neighbor distance of node $n$ \\
10 & let $\varepsilon$ be the width of the predefined area \\
11 & let $d=0$ \\
12 & let $f_{m}$ be the forwarding node of antenna $m$ \\
13 & if $m_{n}==m$ and $a_{n} \leq \varepsilon / 2$ and $d<d_{n}$ then \\
14 & $d=d_{n}$ \\
15 & $f_{m}=n$ \\
16 & end if \\
17 & add $f_{m}$ to $F$ \\
18 & end for \\
19 & return $F$ \\
\hline
\end{tabular}

Since the six directional antennas of node are the same, the full circle is divided into a number of predefined directions which the angles between them are equal. Therefore, it is reasonable that the connections between the adjacent forwarding nodes constitute an approximation of the regular hexagon, then the forwarding nodes become the vertices of hexagon (Figure 4). This hexagon is the basis of HFS.

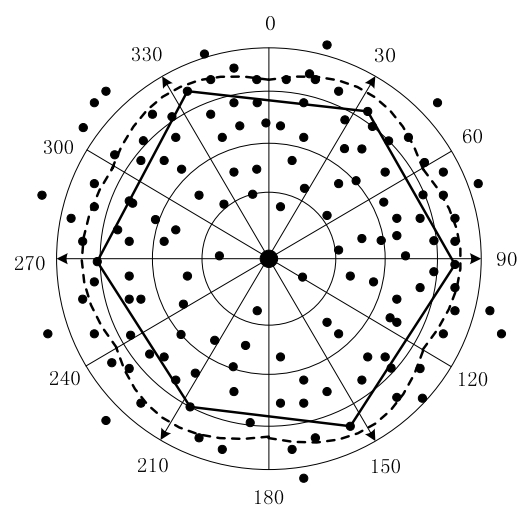

Figure 4. The regular hexagon constituted with the forwarding nodes

\section{Request forwarding strategy}

If the route request packets are flooding among forwarding nodes directly, unnecessary redundancy will be caused too. As shown in Figure 5, when a forwarding node 
receives a new route request packet, it forwards the packet to its all forwarding nodes, then a large number of redundant forwarding are caused.

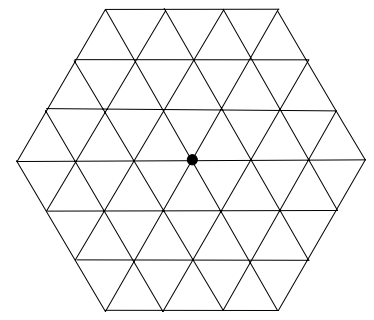

Figure 5. Route request packets are flooding among forwarding nodes

In fact, only a part of the route request forwardings are valuable. So the request forwarding strategy can be improved by reshaping the route request flow pattern such that all of the forwarding nodes receive the same route request packet only once. We remove useless route request forwardings according to the following rules:

- If the number of hops between the sending node and source node is greater than or equal to the number of hops between the receiving node and source node, we remove the route request forward between them.

- If a node receives the same route request packet from more than one node, the forwarding which lies in clockwise direction of the connection between the node and source node will be retained, and the rest are removed.

The reshaped pattern of the route request flow is shown in Figure 6. We can find that, only the source node forwards route request packet to all of its forwarding nodes, other nodes just need to forward route request packet to one or two forwarding node, thus the number of nodes involved in the route discovery process is reduced to a minimum. At the same time, each node only receives route request packets from only one node.

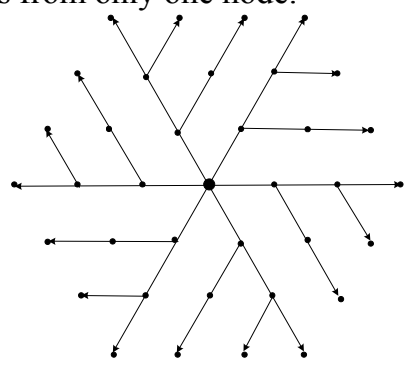

Figure 6 . The pattern of the route request flow

\section{The proposed strategy}

The proposed Hexagonal Flooding Strategy is presented in Algorithm 2. In order to adapt to the algorithm, we have added two fields in the route request packet. The first field is the quantity of selected forwarding nodes of the packet receiving node, which is denoted by $q$, the second field is the number of the antenna that forwards this packet, which is denoted by $m$.

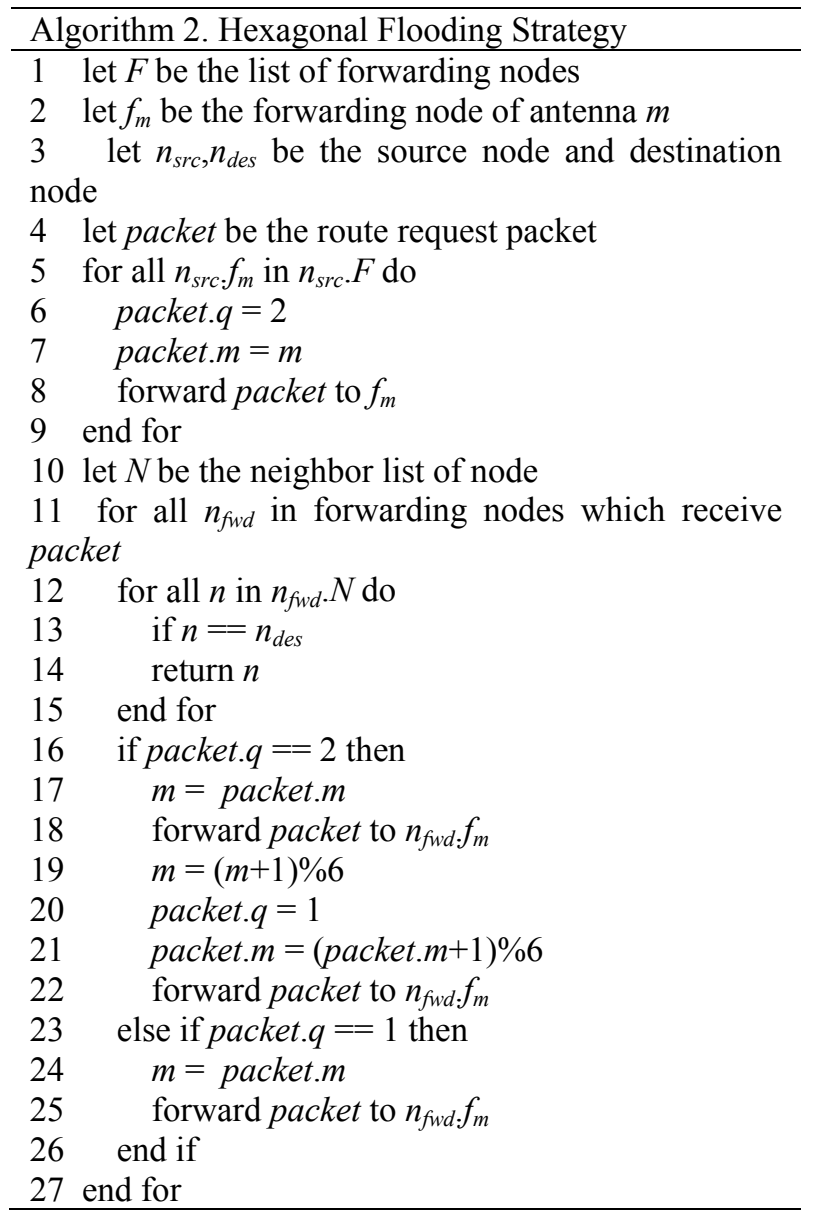

In Hexagonal Flooding Strategy, the source node forwards route request packets to all of its forwarding nodes and let them forward route request packets to two forwarding nodes. Upon receiving the route request packet, each forwarding node checks its own neighbor list to find the destination node. If the destination node exists in its neighbor list, the route from the source node to the destination node is found. Otherwise, the forwarding nodes forward route request packets to its forwarding nodes again. According to the quantity of selected forwarding nodes $\mathrm{q}$, there are two kinds of forwarding behavior as shown in Algorithm 2.

Figure 7 shows a scenario that uses Hexagonal Flooding Strategy. Black node is the source node.

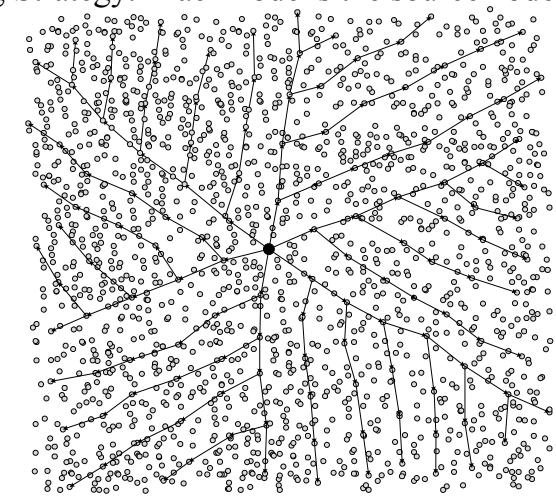

Figure 7. An example of Hexagonal Flooding Strategy 


\section{SIMULATION AND ANALYSIS}

We present experimental results which evaluate our approach in a simulation environment built with NS-2 simulator. Three important performance metrics are evaluated: route discovery success rate, average number of nodes and forwardings involved in the route discovery process. In these simulations, the performance of the proposed Hexagonal Flooding Strategy is compared against the performances of the traditional DSR flooding strategy $^{[2]}$, the probabilistic counter-based method $(\mathrm{PCM})^{[4]}$, the honeycomb strategy ${ }^{[9]}$, and the network density and mobility aware energy-efficient broadcast route discovery strategy $(\mathrm{DMEF})^{[12]}$.

The simulation was performed using NS-2 which assumed an ideal MAC layer. Simulations are repeated 100 times, in which the route request flooding process is initiated once. At the end of each round of simulation, whether the route is found, the number of nodes and the number of forwardings involved in the route discovery process are collected for the purpose of analysis and comparison. The simulation parameters are given in Table 1.

TABLE 1. PARAMETERS For PERFORMANCE Simulation

\begin{tabular}{cc} 
Parameters & Value range \\
Range of simulation environment & $2000 \mathrm{~m} \times 2000 \mathrm{~m}$ \\
Medium access control protocol & IEEE 802.11 \\
Transmission range of nodes & $200 \mathrm{~m}$ \\
Channel capacity & $3 \mathrm{Mbps}$ \\
Processing delay of data packets & $0.01 \mathrm{~s}$ \\
Antenna beamwidth & $\pi / 3$ \\
Simulation times & 100 \\
\hline
\end{tabular}

There are two parameters have important impact on the experimental results: Time To Live (TTL) and the quantity of nodes. So simulations are carried out to evaluate the performance across wide range of TTL and the quantity of nodes. When the simulations are carried out across wide range of TTL, the quantity of nodes are 500; on the contrary, when the simulations are carried out across wide range of the quantity of nodes, the TTL value used was 10 .

\section{A. Simulations across wide range of TTL}

Figure 8 shows the route discovery success rates of five strategies for seven different TTL values. The traditional DSR flooding strategy has the lowest route discovery success rates, the other four strategies have great improvement on route discovery success rates. It can be seen in the figure 9 that HFS and the honeycomb strategy achieve low average number of involved nodes, this is the biggest advantage of directional antenna forwarding strategy. Because the forwardings in HFS are directed, therefore, when TTL is greater than a certain threshold value, the route request packets are forwarded to the network boundary, average number of nodes involved will not continue to increase.

As shown in Figure 10, HFS achieves lower average number of forwardings across wide range of TTL than the honeycomb strategy. This is because the number of forwarding nodes are as small as possible and each node only receives route request packets from only one node. These results show that HFS achieves high route discovery success rate while keeping the number of involved nodes and forwardings low across wide range of TTL.

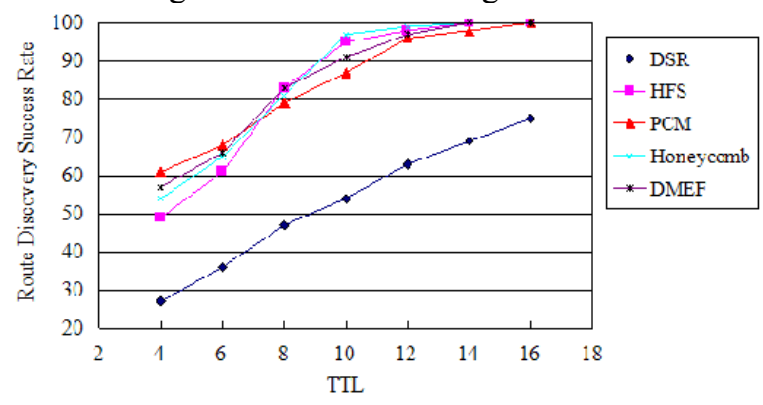

Figure 8 . The route discovery success rate vs TTL

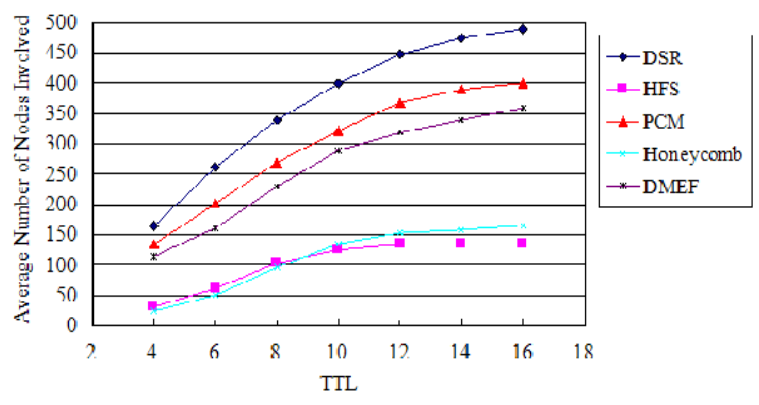

Figure 9. Average number of nodes involved vs TTL

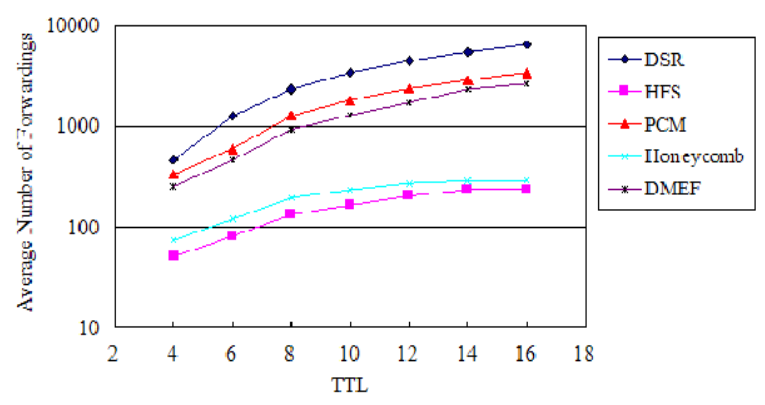

Figure 10. Average number of forwardings vs TTL

\section{B. Simulations across wide range of the quantity of nodes}

Figure 11 shows the route discovery success rate against the quantity of nodes. Similarly, The traditional DSR flooding strategy has the lowest route discovery success rates too. It can be seen in the figure 12 that the average number of nodes involved in HFS does not increase with the increase of the quantity of nodes. This is because the number of forwarding node is mainly dependent on transmission range of nodes and range of network environment, and has no direct relationship with the quantity of nodes. Figure 13 shows a similar result, the average number of forwardings in HFS does not increase with the increase of the quantity of nodes too.

As can be seen from the results across wide range of TTL and the quantity of nodes, HFS can achieve high route discovery success rate while keeping the number of involved nodes and forwardings low. HFS also has its disadvantages, and that is, it can only be used for a particular environment. This is an important trend in the 
research of MANET: the researchers study the performance improvement in specific network environment, rather than improve the performance of generic protocol.

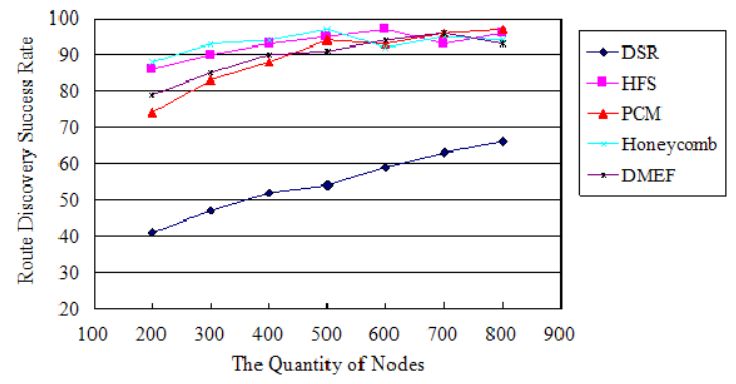

Figure 11. The route discovery success rate vs the quantity of nodes

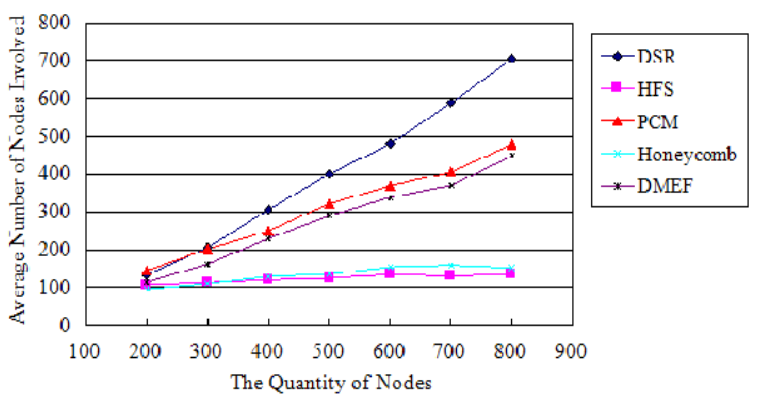

Figure 12. Average number of nodes involved vs the quantity of nodes

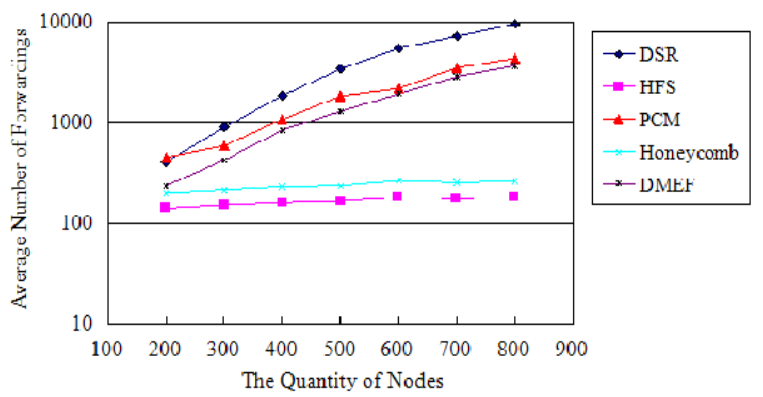

Figure 13. Average number of forwarding vs the quantity of nodes

\section{CONCLUSIONS}

A novel route request flooding approach called Hexagonal Flooding Strategy for Ad hoc networks using directional antennas has been propose in this paper. By constructing a honeycomb pattern, the forwarding nodes which form the honeycomb vertices are selected. The transmissions between forwarding nodes which form the honeycomb edges are reshaped according to certain rules to optimize flow directions. Route request packets are forwarded from source node to the honeycomb vertices along honeycomb vertices. Simulation results across wide range of TTL and the quantity of nodes show HFS can achieve high route discovery success rate while keeping the number of involved nodes and forwardings low.
In future work, we plan to expand the proposed strategy to support more general environment. Some enhancements to the proposed strategy will be included.

\section{ACKNOWLEDGMENT}

Qian Long thanks for being supported by the National Natural Science Foundation of China under Grant No. 61272454 and the Specialized Research Fund for the Doctoral Program of Higher Education of China under Grant No. 20130141110022.

\section{REFERENCES}

[1] D.P. Agrawal, Q.A. Zeng, "Introduction to wireless and mobile systems", Ad Hoc and Sensor Networks, Brooks/Cole-Thomson Learning, pp.297-348, 2003.

[2] Romit Roy Choudhury, Nitin H. Vaidya, "Performance of ad hoc routing using directional antennas", Ad Hoc Networks, vol.3, no.2, pp.157-173, 2005.

[3] D.B. Johnson, D.A. Maltz, Dynamic source routing in ad hoc wireless networks, in: Imielinski, Korth (Eds.), Mobile Computing, vol. 353, Kluwer Academic Publishers, 1996.

[4] Aminu Mohammed, Mohamed Ould-Khaoua, Lewis M. Mackenzie, Colin Perkins, Jamal-Deen Abdulai, "Probabilistic counter-based route discovery for mobile ad hoc networks", Proceedings of the 2009 International Conference on Wireless Communications and Mobile Computing: Connecting the World Wirelessly, pp.13351339, 2009.

[5] J. Abdulai, A. Mohammed, K. S. Nokoe, E. Oyetunji, "Route discovery in wireless mobile ad hoc networks with adjusted probabilistic flooding", Proceedings of the 2009 International Conference on Awareness Science and Technology, pp.99-109, 2009.

[6] Li Jian, Mohapatra, Prasant, "PANDA: A novel mechanism for flooding based route discovery in ad hoc networks", Wireless Networks, vol.12, no.6, pp.771-787, 2006

[7] Yongsuk Park and Taejoon Park, "A Directional Route Discovery Protocol in Ad Hoc Multi-Hop Cellular Networks", IEICE Transactions on Communications, Volume E93.B, Issue 3, pp. 725$728,2010$.

[8] Marco Aurélio Spohn, Jose Joaquin Garcia-Luna-Aceves, "Enhancing the route discovery process of on-demand routing in networks with directional antennas", Lecture Notes in Computer Science, Volume 3042, pp.1000-1011, 2004.

[9] Rully Adrian Santosa, Bu-Sung Lee, Chai Kiat Yeo, "Efficient DSR Route Request Flooding With Directional Antennas", Computer Networks, Volume 54, Issue 13, pp.2293-2309, 2010.

[10] Bani Khalaf M., Al-Dubai A., Buchanan W., "A New Efficient Distributed Route Discovery for Wireless Mobile Ad hoc Networks", Proceedings of the 11th IEEE International Wireless Telecommunications Symposium, pp.1-6, 2012.

[11] Lukas Wallentin, Joachim Fabini, Christoph Egger, Marco Happenhofer, "A cross-layer route discovery strategy for virtual currency systems in mobile ad hoc networks", Proceedings of the 7 th international conference on Wireless on-demand network systems and services, pp.91-98, 2010.

[12] Natarajan Meghanathan, "A Density and Mobility Aware EnergyEfficient Broadcast Route Discovery Strategy for Mobile Ad hoc Networks", International Journal of Computer Science and Network Security, vol.9, no.11, pp.15-24, 2009.

[13] Xiangyang Jin, Thomas Kunz, Ivan Stojmenovic, "Multiretransmission Route Discovery Schemes for Ad Hoc Wireless Networks with a Realistic Physical Layer", Proceedings of the 2012 IEEE 26th International Conference on Advanced Information Networking and Applications, pp.558-565, 2012. 